\section{Academic promotion in Italy}

SIR - In Italy, appointments to tenured positions at the full professor level are not handled through local ad hoc search committees or advertisements in international scientific journals; instead, all vacancies are submitted to the Ministry of University and Scientific Research and Technology (MURST) in Rome, which every four to five years announces a national competition for all disciplines.

This peculiar procedure contributes adversely to the precarious state of health of academic institutions in Italy. For example, in 1988, among the several hundred tenured positions vacant in schools of medicine, five were for professorships in a specialty branch of internal medicine. A committee of five professors in that specialty, with one acting as chairman, evaluated 16 applicants. The committees are asked by state law to ascertain only the "full scientific maturity" of applicants, through examination of their scientific output; there are no other requirements or specific guidelines and the candidates are never interviewed. The discretionary powers of a committee are almost unlimited. It is therefore possible for candidates for a tenured position involving heavy responsibilities and duties in teaching, research and clinical practice to be evaluated without taking into consideration the applicants' previous experience in teaching, clinical skills and proficiency in grant applications. A final decision about the best five was finally reached in November 1989 . The committee's proceedings were available after several months, but there was no mention of the criteria used to evaluate the applicants' "scientific maturity".

We therefore decided to check whether widely applied indicators for evaluation of research based on bibliometric analysis did support the choices of that committee. A search was made on the five successful candidates (winners), and on four rejected applicants (losers) who are especially well thought of in the scientific community.

The MEDLINE database provided the list of all publications from 1966 to 1988 . However, a publication count gives only a measure of the total volume of the research output and does not indicate the quality of the work. Because citation frequency analysis has been increasingly used in the evaluation of science, a search was made of the citations for each candidate. Citations from 1965 to 1988 were obtained from the Science Citation Index (SCI) database for the articles where the candidates appeared as first or last author. This approach overcomes, at least partly, the criticism that in a routine search citations are awarded to

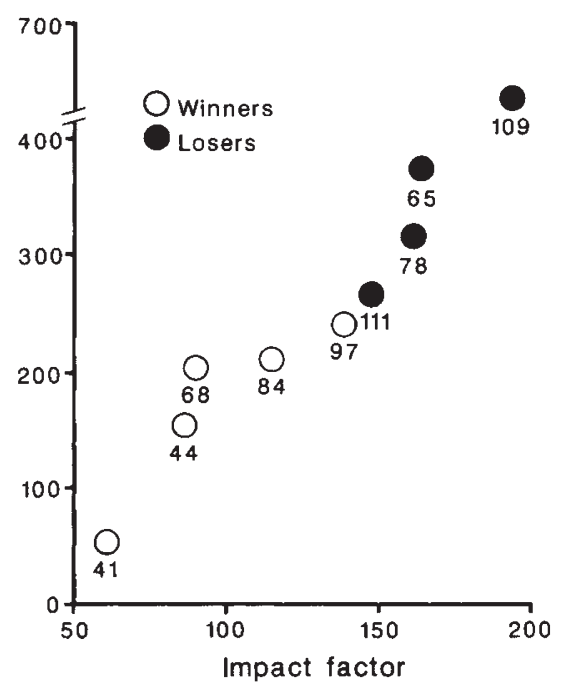

Correlation between cumulative impact factor and number of citations. Under each circle the total number of papers is reported.

the first author. The mean of the citations for the four excluded candidates was $397 \pm 165$, versus $172 \pm 74$ for the winners $(\mathrm{P}<0.01)$.

Another reliable estimate of the performance of scientific papers is their SCI impact factor (IF). The IF is the ratio of the number of citations a journal receives to the number of papers published over a period of time. Furthermore, a paper published in a high-impact journal indicates careful peer review even for controversial ideas. All publications

\section{Biological accord}

SIR - Lennart Philipson's Commentary, "Turmoil in European biology" (Nature 351, 91; 1991), was a timely analysis of the dire straits through which basic biological research in Europe is passing - with little sign of calm water and prosperity ahead.

Part of Philipson's remedy is the formation of a joint coordinating body for all organizations representing biological research in Europe. As it happens, plans to establish such an organization had been under discussion for more than two years, following an initiative taken by Professor Karl Ullrich and $\mathrm{Mr} \mathrm{B}$. Sälzer, MEP, in 1989, and EUSEB (the European Union of Societies for Experimental Biology) officially came into being in Würzburg, Germany, on 1 June 1991. The founder members of EUSEB are the European Cell Biology Organization (ECBO), European Developmental Biology Organization (EDBO), European Neuroscience Association (ENA), European Society of Toxicology (EUROTOX), Federation of European Biochemical Societies (FEBS), Federa- were then rated according to the 1988 IF. The four losers had more articles published in the 15 most-cited journals (15 vs 4). The five winners had a cumulative IF score largely inferior to that of the four losers; the difference between the means of the two groups $(99 \pm 30$ versus $169 \pm 19)$ was statistically significant $(\mathrm{P}<0.005)$. A positive correlation between cumulative IF and citations was observed (see figure). On the basis of our studies, it is therefore unlikely that the four rejected candidates were deemed unsuitable for an academic promotion to full professor because of insufficient "scientific maturity". These international scientific indicators were ignored and the scale of values adopted by the committee is a mystery. However, the names of one or more members of the committee itself often appeared among the multiauthored papers of the winners (for one of the successful candidates this happened in 70 per cent of the articles).

We conclude that the present machinery for academic promotion, besides being artefactual and unreliable, is very much like an 'old boy' network, and has led many of Italy's leading researchers to leave the country. There is an urgent need for new rules and guidelines to be incorporated into the decision-making process of academic promotion.

GIAN Franco GaEtanI ANNA MARIA FERRARIS

University of Genova Medical School, ISMI-Viale Benedetto XV, 6 16132 Genova, Italy

tion of European Pharmacological Societies (EUROPHARM) and Federation of European Physiological Societies (FEPS), with the European Molecular Biology Laboratory (EMBL) as an associate member.

Because, as far as we are aware, no registry of all the federations of European biological societies exists, as one of EUSEB's first public activities, we invite the secretaries of all such federations to contact Professor Guy Dirheimer, Institut de Biologie Moleculaire et Cellulaire du CNRS, 15 rue René-Descartes, 67084 Strasbourg Cedex, France.

The aims of EUSEB are very much in line with those outlined in Philipson's Commentary, and include in particular the establishment of contacts with national and supranational governmental and private institutions at the highest level, with a view to seeking solutions to the problems confronting basic biological scientists in all disciplines.

HAMISH M. KEIR

(President, EUSEB)

Department of Molecular

and Cell Biology,

University of Aberdeen,

Aberdeen AB9 1AS, UK 